\title{
Performance and Technical Challenges of Liquid Argon Detectors
}

\author{
Brian Rebel
}

MS 220, Fermilab, Batavia, IL 60510, USA

\begin{abstract}
Liquid argon time projection chambers offer the possibility of incredible resolution of particle interactions. This review outlines the ongoing research and development towards the realization of a multi-kiloton scale detector. The ICARUS and ArgoNeuT experiments which make use of liquid argon time projection chamber technology are also described.
\end{abstract}

Keywords: Neutrino, Liquid Argon

PACS: $07.05 . \mathrm{Fb}$

\section{LIQUID ARGON TIME PROJECTION CHAMBERS}

Liquid argon time projection chambers (LArTPCs) offer the possibility of imaging charged particle interactions with incredible spatial resolution. A charged particle passing through liquid argon will ionize the argon liberating $\approx 55,000$ electrons per centimeter. The ionization electrons are drifted through an electric field towards readout wires where they produce current that is digitized by the readout electronics. The typical resolution for a LArTPC is on the order of $0.05 \mathrm{~cm}$, providing true images of the particle interactions. This fine grained resolution is important for a variety of physics measurements, including neutrino oscillations, proton decay and neutrino cross-sections.

This review will present an overview of the state of research and development efforts underway that are designed to make the realization of a multi-kiloton scale LArTPC possible. It will also report the progress of two recent experiments, ICARUS and ArgoNeuT, that have taken data in neutrino beams.

\section{CURRENT RESEARCH AND DEVELOPMENT EFFORTS}

\section{Purification of the Liquid Argon}

Although LArTPC technology is very promising, much research and development must be done to make it viable for multi-kiloton scale detectors. One of the primary concerns in operating LArTPCs is the removal of electronegative contaminants from the liquid argon that would prevent the ionization electrons from reaching the readout wires. Purification of the liquid argon is necessary for any LArTPC to function, but it is especially im- portant for LArTPCs that drift electrons over several meters, as would be the case in multi-kiloton sale detectors.

One aspect of purification is knowing which materials are more likely to poison the liquid argon and drive down the free electron lifetime in the liquid. Fermilab has a dedicated materials test stand (MTS) to test possible materials for use in LArTPCs. This test stand is a 250 liter capacity cryostat that has an airlock that allows materials to be placed in the cryostat without first emptying it of the liquid. A sample cage is located in the airlock to hold materials that are to be tested. The cage can be positioned at any depth in the cryostat allowing measurements to be made at a variety of temperatures. An internal filter located in the cryostat purifies the liquid in situ, and a condenser collects the boiled off argon gas so that it can be liquified and returned to the bulk volume for closed operation of the system. In addition to determining which materials are safe to put into LArTPCs, the MTS has also identified a relationship between the concentration of water in the liquid and the electron lifetime. Water appears to be the primary contaminant that degrades electron lifetime. It is interesting to note that water contamination appears to only adversely affect the lifetime when it is in the warm vapor phase of the LArTPC where temperatures are greater than 150K. Materials that are completely submerged in the liquid argon or near the liquid-gas boundary do not outgas water [1]. These results are shown in Fig. 1.

Another test stand is being built at Fermilab to determine whether evacuation of the cryostat is necessary to achieved electron lifetimes of the magnitude necessary to operate multi-kiloton scale detectors. That project is known as the Liquid Argon Purity Demonstrator (LAPD). Evacuation is one of the traditional steps in achieving high purity inside LArTPCs. The evacuation removes water from the surfaces inside the cryostat, however it is expensive to make evacuable cryostats the necessary size for multi-kiloton scale detectors. In fact, 


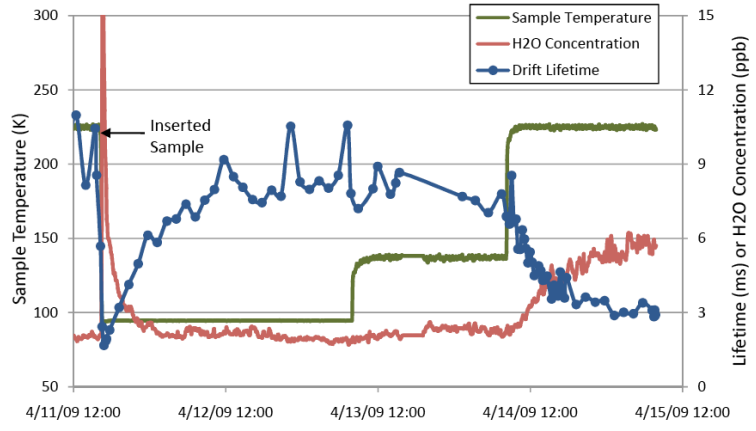

FIGURE 1. Material sample temperature, water concentration inside the cryostat, and electron lifetime as a function of time.

evacuation can increase the cost of the cryostat by more than a factor of two. Instead of evacuating, the LAPD will cycle several volumes of gaseous argon through its cryostat before filling with liquid. The argon will push the ambient atmosphere out of the vessel, reducing the oxygen contamination to the level of 100 parts per million (ppm) or less. At that point the gaseous argon will be forced through the filter system to enable a more efficient removal of the water and oxygen contamination. Once the contamination is at an acceptable level, $50 \mathrm{ppm}$ or less, liquid argon will be introduced into the system and it will also undergo continuous recirculation through the filters. The ultimate goal is to reduce the contamination to a level of less than 1 part per billion. The procedure will be declared a success if the electron lifetime required to drift at least $80 \%$ of the ionization electrons a distance of $2.5 \mathrm{~m}$ can be achieved, that is a lifetime of $5 \mathrm{~ms}$ or more.

The initial tests with LAPD will be performed using an empty vessel. No materials apart from the devices to measure the electron lifetime will be in the vessel. In the second phase of the program, materials that would be used in an LArTPC will be placed in the vessel and the test repeated. If the necessary electron lifetime of $5 \mathrm{~ms}$ can be achieved in this test, then LAPD will have shown that evacuation is not a requisite step in running an LArTPC. In addition to these studies, LAPD will also help determine how to scale the cryogenic system to the size necessary for a multi-kiloton detector. It will study the concentration of oxygen at various depths in the tank during the purge with gaseous argon. It will also record the number of liquid argon volume exchanges necessary to reach the required drift time as well as the rate of exchange needed to maintain that lifetime and the filter capacity as a function of flow rate. Finally, it will also show how possible it is to recover from a contamination of a known amount of oxygen and water.

\section{CRYOSTAT TECHNOLOGY}

If LAPD is successful, it will show that the next generation of LArTPCs will not need to be evacuated, allowing for the use of novel cryostat technologies. One option is the use of a membrane cryostat. The membrane cryostat contains the liquid argon using sheets of approximately $0.3 \mathrm{~cm}$ stainless steel that are welded together to form a leak tight seal. The sheets have corrugations in them to allow for differential contraction during the cool down phase of filling the cryostat. The membrane is then surrounded by several layers of insulation, fluid containment and structural members, with the ultimate structural support coming from concrete surrounding the cryostat. This technology has been used for decades to transport liquid natural gas in tanker vessels. This cryostat technology is under consideration for the Long Baseline Neutrino Experiment, LBNE.

\section{READOUT SYSTEMS}

There is ongoing effort to develop a readout alternative to the traditional wire planes immersed in liquid argon. One possible system is the large electron multiplier (LEM) that offers several advantages over traditional systems [2]. The LEM operates by extracting the ionization electrons from the liquid argon and drifting them through the gas phase to the LEM device. The device itself is copper clad FR4 that is approximately $1 \mathrm{~mm}$ thick with $1 \mathrm{~mm}$ diameter holes spaced $1 \mathrm{~mm}$ apart. The electrons are drifted through the holes in a high electric field and undergo multiplication as they traverse the holes. This multiplication produces a signal to noise ratio of 800/10 [2], a significant gain over traditional systems. These devices are also more robust than the traditional wires with less chance of a channel breaking due to mechanical stress while filling the volume with liquid argon.

\section{STATUS OF ICARUS AND ARGONEUT}

ICARUS, containing 600 tons of liquid argon and located in the Gran Sasso Laboratory, is the largest detector in the world using LArTPC technology and is the culmination of 20 years of development. The program to produce a large detector started with a 501 test stand exposed to the WANF neutrino beam at CERN and included steps at 3 tons and 300 tons as well. The ICARUS detector is composed of 2 modules, each containing 300 tons of liquid argon and was filled May 18, 2010. It is currently taking data using the CNGS neutrino beam. ICARUS has already observed several neutrino interactions from the CNGS beam, an example of which is shown in Fig 2. It has also released information on the TPC performance 


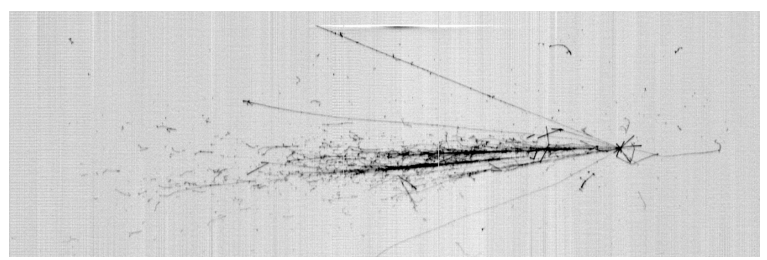

FIGURE 2. Example neutrino interaction in the ICARUS detector. The beam direction is from right to left in this figure.

with respect to electron lifetimes. Both modules have achieved sufficient purity for sufficient ionization electrons to reach the readout wires with good signal to noise ratios. The west module has an equilibrium lifetime of $5.39 \mathrm{~ms}$ and the east module has an equilibrium lifetime of $2.73 \mathrm{~ms}$. The difference is due to different production of impurities in each module, with the source of the difference still under investigation [3].

ArgoNeuT is the first LArTPC to be placed in a low energy neutrino beam, namely the NuMI beam at Fermilab. The ArgoNeuT detector, shown in Fig. 3, was placed in the MINOS hall in December 2008, and was filled with liquid argon on May 8, 2009. It took data through February 2010, and was subsequently removed from the MINOS hall. ArgoNeuT has a capacity of 5001 of liquid argon, with 1751 enclosed inside the TPC. ArgoNeuT has taken both neutrino and anti-neutrino data. In neutrino mode, the beam composition is $91.7 \%$ muon neutrinos, $7.0 \%$ muon anti-neutrinos and $1.3 \%$ electron neutrinos and anti-neutrinos. The beam has a peak energy of $3.3 \mathrm{GeV}$ with a long high energy tail out to $120 \mathrm{GeV}$. The anti-neutrino beam composition is $39.9 \%$ muon antineutrinos, $58.1 \%$ muon neutrinos and $2.0 \%$ electron neutrinos and anti-neutrinos. The peak energy of the beam is still $3.3 \mathrm{GeV}$, with the neutrinos making up the bulk of the high energy tail. Muon anti-neutrinos are the dominant component of the beam for energies less than $5 \mathrm{GeV}$.

The ArgoNeuT collaboration is still analyzing its data. It expects to have 6600 charged-current muon neutrino interactions in its dataset, along with 4900 chargedcurrent muon anti-neutrino interactions. Of those interactions, 600 are expected to be quasi-elastic muon neutrino interactions. In addition, there are approximately 130 charged-current electron neutrino interactions.

Much effort has gone into developing a fully automated reconstruction and simulation framework for use by ArgoNeuT as well as future LArTPCs. The software framework is known as LArSoft [4]. It has been used to develop particle identification techniques based on the energy deposition of particles in the liquid argon. The procedure looks promising, allowing the collaboration to separate proton tracks from those caused by muons and pions.

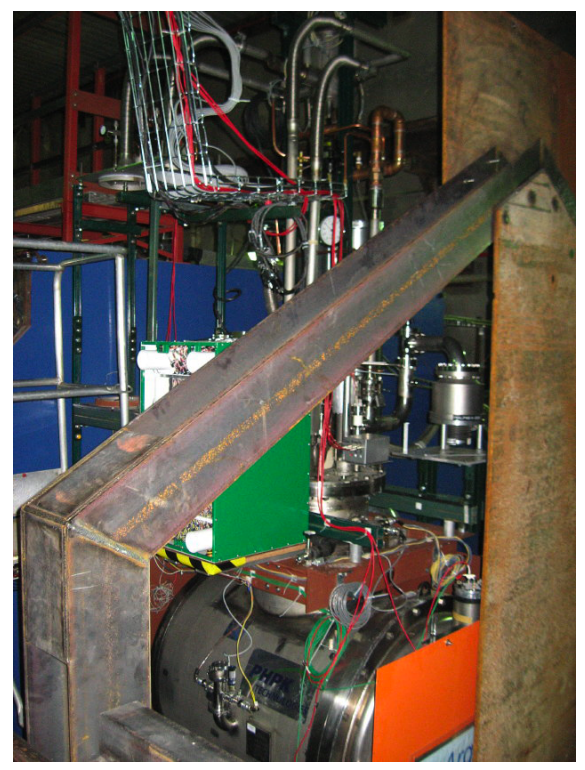

FIGURE 3. The ArgoNeuT cryostat.

\section{OUTLOOK}

Many test stands around the world are pushing LArTPC technology towards a kiloton-scale detector. Significant progress has been made in understanding how to keep the liquid argon free from electronegative contaminants. New readout technology advances are being made with the development of the LEM readout, which offers significant improvements in the signal to noise ratio compared to traditional wire readout. Additionally, new cryostat technologies are being explored.

ICARUS and ArgoNeuT have data from neutrino beams and the first results from these experiments are eagerly anticipated.

\section{REFERENCES}

1. Andrews, R. et al. 2009 Nucl. Instrum. Meth. $\mathbf{A 6 0 8} 251$

2. Badertscher, A. et al. 2010 Nucl. Instr. Meth. A617 188

3. Sala, P. 2010 presented at NOW 2010

4. https://cdcvs. fnal.gov/redmine/projects/larsoftsvn 